\title{
Development and In Vitro Characterization of Microemulsions of Isotretinoin
}

\author{
Aslı Gürbüz ${ }^{1}$, Sevgi Güngör ${ }^{1}$, Meryem Sedef Erdal ${ }^{1 *}$ \\ IIstanbul University, Faculty of Pharmacy, Department of Pharmaceutical Technology, 34116
}

\begin{abstract}
Microemulsions are nano-sized colloidal drug carriers which offer several advantages such as ease of preparation, thermodynamic stability, high solubilizing capacity for both of lipophilic and hydrophilic drugs and penetration enhancement. The aim of this study was to prepare novel microemulsions of isotretinoin, a highly lipophilic anti-acne drug, for its topical application. The pseudo-ternary phase diagrams were constructed at different oil to surfactant/co-surfactant mixture using isopropyl myristate (oil phase), Labrasol (surfactant), Kolliphor HS15, Kolliphor EL or Plurol Oleique CC497 (co-surfactant) and water. The physicochemical properties and storage stability of microemulsions were investigated. The developed microemulsions were characterized in terms of isotropy, particle size and size distribution, $\mathrm{pH}$, refractive index, rheological behaviour, and conductivity. Spherical shape and droplet size of microemulsions were supported by transmission electron microscopy (TEM). Optimized formulations were found to be physically stable over a period of six months. In conclusion, microemulsions could be promising colloidal carriers for topical delivery of isotretinoin.
\end{abstract}

Keywords: microemulsion, colloidal systems, isotretinoin, topical drug delivery

\section{INTRODUCTION}

There has been increased interest during recent years in the use of topical vehicles that may modify drug penetration into the $\operatorname{skin}^{1}$. The most difficult aspect in skin delivery of drugs is to overcome the barrier of stratum corneum. Various strategies have been employed to achieve delivery of drugs into skin. Among these strategies, microemulsions have been suggested to serve as efficient promoters of drug localization to skin ${ }^{2-4}$.

Microemulsions are thermodynamically stable, fluid and isotropic colloidal nanocarriers with a dynamic microstructure that form spontaneously by combin-

${ }^{\star}$ Corresponding Author: Meryem Sedef Erdal

E-Mail address: serdal@istanbul.edu.tr 
ing appropriate amounts of oil, water, surfactant and a co-surfactant ${ }^{5-7}$. They are prepared mostly by the phase titration method and can be depicted with the help of pseudo-ternary phase diagrams. Some of the potential mechanisms by which microemulsions would improve transport of drugs to the skin are described below: ${ }^{13,3,8-10}$

- Ingredients of microemulsions can modify the diffusional barrier of the stratum corneum either by perturbation/fluidization of intercellular lipid bilayers or denaturation of intracellular keratin or modification of its confirmation.

- Due to the high solubilization capacity of microemulsions, both for the hydrophilic and lipophilic drugs, an increased concentration gradient towards the skin can be reached.

- The ultralow interfacial tension and the continuously fluctuating interfaces of microemulsions can facilitate drug penetration into deeper skin layers compared to conventional formulations.

- The partitioning and solubility of drugs in stratum corneum could be increased depending on microemulsion composition.

- The internal phase can act as a drug reservoir resulting controlled and sustained release from microemulsions.

In view of all these features of microemulsions, the present study aims to explore microemulsions as alternative topical carriers for isotretinoin, with an objective to facilitate skin targeting of the drug while decreasing its systemic exposure and toxicity. For that purpose the pseudo-ternary phase diagrams of microemulsion systems were constructed at different surfactant/co-surfactant ratios using isopropyl myristate (IPM) as oil phase, Labrasol as surfactant and Kolliphor HS15 (KHS), Kolliphor EL (KEL) or Plurol Oleique CC497 (PLO) as co-surfactant. It has been reported that IPM enhances skin permeation by acting as a fluidizer of intercellular lipids and affects the lipid rich phase in the stratum corneum, thereby reducing its barrier function ${ }^{11}$. Labrasol is a surfactant which has been shown to significantly enhance the permeation of lipophilic drugs through the skin ${ }^{12}$. The co-surfactants KHS, KEL and PLO served frequently as penetration enhancers in the scientific literature ${ }^{13-17}$. The physicochemical properties such as droplet size, refractive index, electrical conductivity, $\mathrm{pH}$, and rheology of the microemulsions were measured and TEM analysis was performed. Optimized formulations were found to be stable over a period of six months at $25^{\circ} \mathrm{C}$ $\pm 2^{\circ} \mathrm{C}$ and $60 \% \pm 5 \%$ relative humidity (RH). 


\section{METHODOLOGY}

\section{Materials}

Isotretinoin, polyoxyl 35 castor oil (KEL) and polyoxyl 15 hydroxystearate (KHS) were kind gifts of BASF (Limburgerhof, Germany). IPM was purchased from Sigma (St. Louis, MO, USA). Caprylocaproyl macrogol-8 glycerides (Labrasol) and polyglyceryl-3 oleate (PLO) were kindly provided by Gattefossé (Lyon, France). All other chemicals and reagents used were of analytical grade.

\section{Construction of Pseudo-ternary Phase Diagrams}

Pseudo-ternary phase diagrams were constructed to determine the appropriate concentration range of components necessary for the formation of microemulsions prepared with the water titration method at ambient temperature. IPM (oil phase) to surfactant/co-surfactant mixture ratio varied from 1:9 to 9:1 (w/w). Based on pre-formulation study data, the mixing ratios of surfactant/ co-surfactant $\left(\mathrm{K}_{\mathrm{m}}\right)$ were fixed as $4: 1$ and $3: 1$. The mixture of oil and surfactant/ co-surfactant at predetermined weight was titrated drop wise with water under moderate magnetic stirring, at ambient temperature. Following each addition, the mixtures were stirred and then allowed to equilibrate. After equilibration, they were visually assessed for phase separation, transparency and flow properties. Transparent, homogenous (single-phase) and, low viscous systems were considered as microemulsion ${ }^{18}$. Titration was stopped with the presence of a cloudy system and/or phase separation. The quantity of the aqueous phase required to make the mixture turbid was recorded. Based on the phase diagrams, appropriate concentration of components were chosen and used in the preparation of drug loaded microemulsions. Drug loaded microemulsions were prepared as follows: Isotretinoin (0.05\%) was weighed into a small glass vial and dissolved in required quantity of IPM under magnetic stirring. Appropriate amount of surfactant/co-surfactant was added to oil phase and was mixed to yield a homogenous solution. The solution was titrated with water up to $100 \%$ (w/w) under magnetic stirring and the obtained microemulsion systems were allowed to equilibrate at ambient temperature. Drug loaded microemulsions were stored in well closed amber coloured vials at room temperature, and protected from light due to the very poor photostability of isotretinoin.

\section{Characterization of Microemulsions}

\section{Droplet Size Measurements}

The droplet size and polydispersity index (PDI) values of plain and isotretinoin loaded microemulsions were determined at $25^{\circ} \mathrm{C}$ with permanent angle of $173^{\circ}$ by a Zeta Sizer (Nano ZS, Malvern Instruments, UK) without dilution with water 
to avoid phase separation ${ }^{17}$. All samples were analyzed in triplicates after prefiltering ( $0.45 \mathrm{~mm}$, Millex, Merck Millipore, Billerica, MA, USA). The droplet size was expressed as average size of droplets in the system and PDI indicated the width of the size distribution ${ }^{19}$.

\section{Microscopic Analysis}

\section{Polarized Light Microscopy}

Microemulsion formulations were examined under a polarized light microscope (Olympus BX51 U-AN 360, Tokyo, Japan) in order to verify their isotropic nature. A drop of the freshly prepared microemulsion was placed between a coverslip and a glass slide and observed under cross-polarized light. It is expected that an isotropic material, such as a microemulsion, will not interfere with the polarized light and the field of view will remain dark ${ }^{20}$.

\section{Transmission Electron Microscopy}

Transmission electron microscopy (TEM) was used to characterize the morphology of the microemulsions ${ }^{21}$. For this purpose, a microemulsion drop was directly deposited on a carbon-coated copper grid and allowed to dry for $60 \mathrm{~min}$ at room temperature. Then, the grid stained with one drop of $2 \%$ (w/w) phosphotungstic acid, excess of the solution was removed with a filter paper and allowed to dry for $5 \mathrm{~min}$ before examination under the electron microscope (JEM-1011, JEOL, Japan).

\section{Electrical Conductivity Measurements}

The electrical conductivity of plain and drug loaded microemulsions were measured with a conductometer (EuTech PC 700; Eutech Instruments, Landsmeer, the Netherlands) at room temperature.

For the assessment of the microstructure $3 \mathrm{~mL}$ of the IPM/(Labrasol/KEL) mixture at ratio 1:9 was titrated by water stepwise and at each step, $1 \mathrm{~mL}$ of sample was used for the measurement of the electrical conductivity at room temperature $^{22}$. The evaluation was made by plotting the conductivity values $(\kappa)$ versus the water percentages $\left(\varphi_{\mathrm{w}}\right)$ obtained experimentally and the percolation thresholds were determined from the peaks of the plot. The measurements were carried out in triplicate, and results were presented as mean \pm SD.

\section{Rheology}

Rheological measurements (shear stress, shear rate and apparent viscosity) were performed using a cone and plate Brookfield Rheometer (Brookfield DV3THACJo, Middleboro, MA, USA) in triplicate in a temperature controlled environment at $25^{\circ} \mathrm{C}$ and rotational speed was ranged from 10-100 rpm. 


\section{pH}

The $\mathrm{pH}$ values of plain and drug loaded microemulsions were measured by direct immersion of $\mathrm{pH}$ meter electrode (EuTech PC 700; Eutech Instruments, Landsmeer, the Netherlands) in the formulations at room temperature. Before each measurement calibration was performed using standard buffer solutions of $\mathrm{pH}$ 4.0, 7.0, and 10.0, respectively. The measurements were carried out in triplicate, and results were presented as mean $\pm \mathrm{SD}$.

\section{Refractive Index}

The refractive index values of the plain and drug loaded microemulsion formulations were measured by an Abbe refractometer (Atogo Co., Ltd, Tokyo, Japan) by placing one drop of the microemulsion sample on the slide at room temperature. The measurements were carried out in triplicate at $25^{\circ} \mathrm{C}$.

\section{Evaluation of Stability}

\section{Centrifugation}

Microemulsions were centrifuged (Hettich Zentrifügen D-7200) at $15000 \mathrm{rpm}$ for $30 \mathrm{~min}$ at ambient temperature to assess the thermodynamic stability. The formulations that did not show any phase separation and cloudy appearance after centrifugation were taken for freeze-thaw cycle.

\section{Freeze-thaw cycle}

Freeze-thaw cycles were performed by freezing the microemulsions at $-20^{\circ} \mathrm{C}$ for $12 \mathrm{~h}$ followed by thawing at $25^{\circ} \mathrm{C}$ for $12 \mathrm{~h}$. This process was repeated two times for each sample. Then the samples were examined for clarity, phase separation and droplet size.

\section{Storage stability}

The storage stability of plain microemulsions was followed according to ICH Q1 (R2) at $25 \pm 2{ }^{\circ} \mathrm{C}$ and $60 \% \pm 5 \%$ relative humidity (RH) up to 6 months ${ }^{23}$. The physicochemical parameters include appearance and droplet size and its distribution were determined.

\section{Statistical Analysis}

The statistical analysis was performed using one-way analysis of variance. A multiple comparison test was used to compare different microemulsion formulations and $\mathrm{p}<0.05$ was considered as level of significance (GraphPad Prism Software, La Jolla, CA, USA). 


\section{RESULTS AND DISCUSSION}

The main goal of the topical therapy is to target the drug to viable epidermis and upper dermis, by minimizing systemic absorption. However, due to its high lipophilic character (logP: 5.01), isotretinoin tends to accumulate on the skin surface and in the upper stratum corneum, thus its penetration into the lower layers is limited, which restricts the efficiency of topical treatment ${ }^{24}$. Nano-sized colloidal carriers such as microemulsions are considered appropriate carriers due to the increment of partitioning and solubility of drug in stratum corneum, enhancement of thermodynamic activity of drug in the vehicle and/or increasing the permeability of skin ${ }^{8,25}$. Most of the studies demonstrate that more pronounced drug deposition in skin layers rather than percutaneous permeation can be obtained with microemulsions ${ }^{3,4,9}$. Taking all these into consideration, microemulsion type colloidal carriers of isotretinoin were developed and characterized with the aim to increase the dermal penetration of the drug.

\section{Construction of Pseudo-ternary Phase Diagrams}
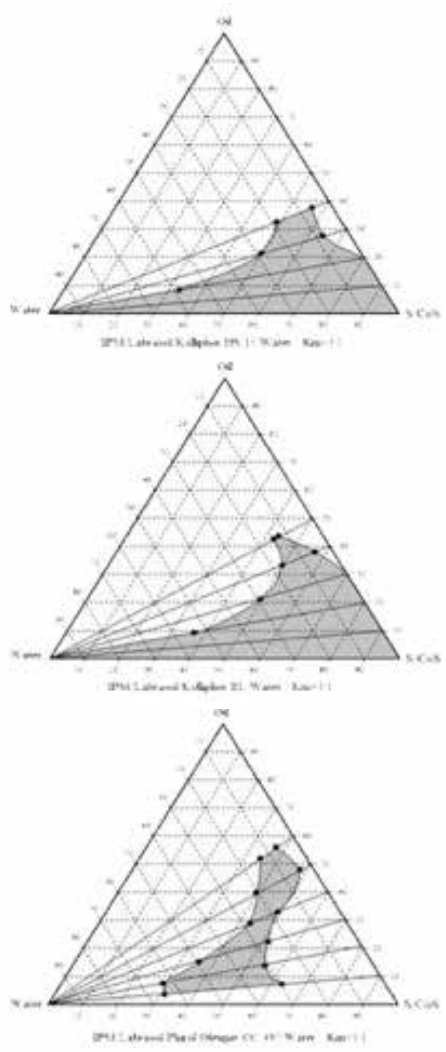
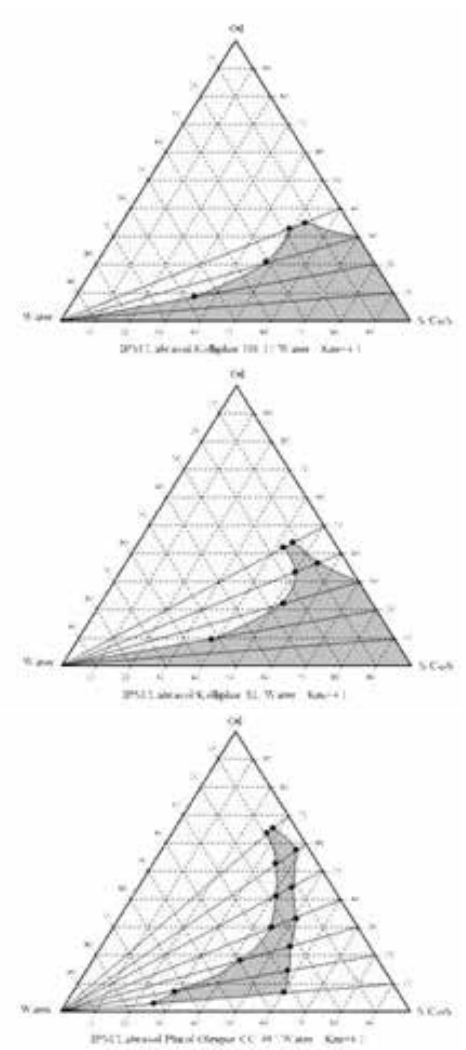

Figure 1: Pseudo-ternary phase diagrams of microemulsion systems containing IPM as oil, Labrasol as surfactant, Kolliphor HS 15 or Kolliphor EL or Plurol Oleique as cosurfactant for $\mathrm{Km}=3: 1$ and $\mathrm{Km}=4: 1$ 
The microemulsion region can be shown graphically in pseudo-ternary phase diagrams, as ratios between oil, water and a fixed mixture of surfactant/co-curfactant ${ }^{26}$. The phase diagrams of the prepared microemulsions with $\mathrm{K}_{\mathrm{m}}$ 4:1 and 3:1 are shown in Fig. 1. Water titration method was used to obtain the components and their concentration ranges that can result in large existence area of microemulsion ${ }^{19,27}$. A large microemulsion area in the phase diagram is usually attributed to the progressive reduction of the interfacial tension and indicates the positive effect of surfactant and co-surfactant on the phase properties ${ }^{18}$.

The water dilution lines representing an increase of water content while decreasing oil, surfactant and co-surfactant levels were plotted on the phase diagrams. The shaded areas were identified as microemulsion areas and the remaining region of the phase diagram represents turbid and conventional emulsions based on visual observation. The area of isotropic microemulsion region changed slightly in size with increasing ratio of surfactant/co-surfactant. The composition of the prepared microemulsion systems consist of IPM (oil phase), Labrasol (surfactant) and KHS, KEL or PLO (co-surfactant) is given in Table 1.

Table 1: Composition of the optimized microemulsion formulations.

\begin{tabular}{|c|c|c|c|c|c|c|c|}
\hline CODE & S:CoS & IPM (\%) & Labrasol (\%) & KHS (\%) & KEL (\%) & PLO (\%) & Water (\%) \\
\hline ME-KHS1 & $3: 1$ & 5.50 & 37.50 & 12.50 & & & 44.44 \\
\hline ME-KHS2 & $3: 1$ & 3.80 & 26.25 & 8.75 & & & 61.14 \\
\hline ME-KHS3 & $4: 1$ & 5.50 & 40 & 10 & & & 44.44 \\
\hline ME-KHS4 & $4: 1$ & 3.80 & 28 & 7 & & & 61.14 \\
\hline ME-KEL1 & $3: 1$ & 5.50 & 37.50 & & 12.50 & & 44.44 \\
\hline ME-KEL2 & $3: 1$ & 3.80 & 26.25 & & 8.75 & & 61.14 \\
\hline ME-KEL3 & $4: 1$ & 5.50 & 40 & & 10 & & 44.44 \\
\hline ME-KEL4 & $4: 1$ & 3.80 & 28 & & 7 & & 61.14 \\
\hline ME-PL01 & $3: 1$ & 6.50 & 43.88 & & & 14.63 & 34.94 \\
\hline ME-PLO2 & $3: 1$ & 5.50 & 37.50 & & & 12.50 & 44.44 \\
\hline ME-PL03 & $4: 1$ & 6.50 & 46.80 & & & 11.70 & 34.94 \\
\hline ME-PLO4 & $4: 1$ & 5.50 & 40 & & & 10 & 44.44 \\
\hline
\end{tabular}

S: Surfactant, CoS: Co-Surfactant

Depending on the physicochemical properties of a drug, different types of microemulsions can be the optimal carrier. Therefore, it is necessary to find the appropriate composition and concentration of components to maximize the drug delivery efficacy of microemulsions ${ }^{9}$. The oil phase, surfactant and co-surfactants for developing isotretinoin loaded microemulsions were selected on the basis of the existence of the microemulsion area and water solubilization capacity. IPM 
is among the most frequently selected components of the oil phase in microemulsions. Water solubilization capacity of IPM is reported to be the highest among various oils, such as oleic acid, used in microemulsion formulation ${ }^{28}$.

In our study, the quantity of isotretinoin to be loaded to the microemulsion formulations has been kept equivalent to its commercial topical formulation (Isotrexin Gel, 0.05\%) and the choice of oil, surfactant and co-surfactants was based on the ability of these components on a stable, skin compatible microemulsion formation with sufficient water content but rather drug solubility ${ }^{9,22}$. When choosing components for microemulsions, it is also important to balance solubility/permeation properties with toxicological considerations ${ }^{26}$. All of the formulated microemulsions were considered as safe regarding the in vitro cytotoxicity study which has been published previously ${ }^{24}$.

\section{Characterization of Microemulsions}

\section{Droplet Size Measurements}

The effect of microemulsion droplet size and large surface area/volume ratio on drug transport into the skin has been shown with several studies'. In our study, the mean droplet diameter of plain and drug loaded microemulsions were found in the range of $1.38 \pm 0.01-9.49 \pm 0.09 \mathrm{~nm}$ and $1.40 \pm 0.03-9.56 \pm 0.14 \mathrm{~nm}$, respectively (Fig. 2). There is no significant difference in average droplet size observed after loading isotretinoin ( $p>0.05$ ). It has been found that increasing water content lead to a decrease in microemulsion droplet size. The formulations containing PLO as co-surfactant (ME-PLO1 - ME-PLO4) presented the highest average droplet size and the droplet diameter increased with the increasing oil and surfactant content. Microemulsion formulations ME-KHS2, ME-KHS4, ME-KEL2 and ME-KEL4 containing KHS and KEL as co-surfactants presented the lowest average droplet size with decreased concentrations of oil and surfactant/co-surfactant mixture.

Polydispersity index (PDI) is an important parameter in the characterization of colloidal drug carriers since it reflects the physical stability of the system and provides information about homogeneity of the samples ${ }^{29,30}$. The PDI values lower than 0.4 indicated homogenous microemulsion systems with narrow size distribution in our study. Incorporation of isotretinoin did not affect the PDI of the microemulsions significantly $(p>0.05)$. 


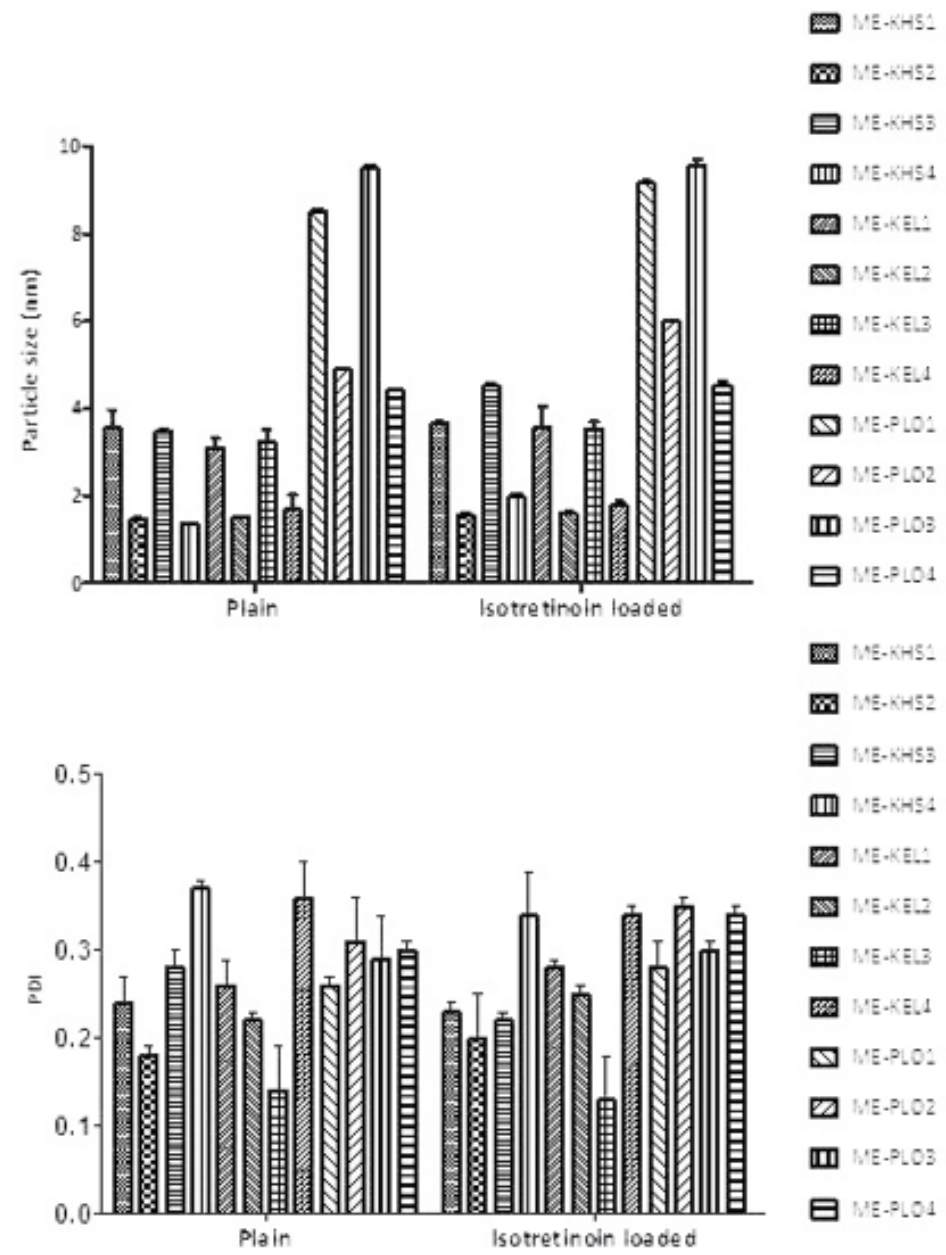

Figure 2: Droplet size and PDI of the plain and isotretinoin loaded microemulsions

\section{Microscopic analysis}

It is well known that various structures, such as liquid crystals, can be formed by microemulsion formulation depending on the components and their concentra$\operatorname{tion}^{17}$. Also the incorporation of the drug can affect the microemulsion structure. Cross-polarized light microscopy is a suitable method for differentiating liquid crystals. Under cross-polarized light microscopy, birefringence can be observed for lamellar and hexagonal liquid crystals but no birefringence is observed for microemulsions ${ }^{31}$. In our study, the completely dark appearance under the polarized light microscope confirmed the isotropic nature of the prepared microemulsions. The TEM images of one formulation (ME-KEL3) are shown in Fig. 3 , which prove that the microemulsion possessed homogeneous and spherical droplets. 

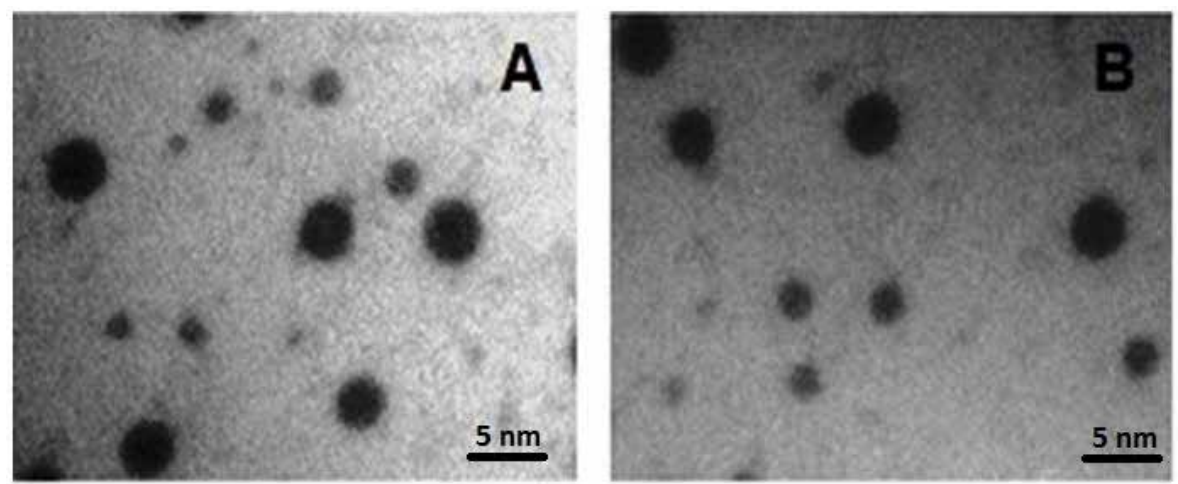

Figure 3: Transmission Electron Microscopy images of A) plain and B) drug loaded microemulsion formulation ME-KEL3

\section{Electrical Conductivity}

The electrical conductivity of plain microemulsion formulations was in the range of $7.50 \pm 0.06-77.65 \pm 0.21 \mathrm{mS} / \mathrm{cm}$ (Table 2) and increased by the increasing amount of water. The increase in conductivity might be caused from the increase in dissociation of surfactant (Labrasol) as a function of water content ${ }^{11,32}$.

Table 2: Conductivity, viscosity, $\mathrm{pH}$ and refractive index of the plain and isotretinoin loaded microemulsions

\begin{tabular}{|l|l|l|l|l|l|l|l|l|}
\hline \multirow{2}{*}{ Code } & \multicolumn{2}{l}{ Conductivity $(\mu \mathrm{S} / \mathrm{cm})$} & \multicolumn{2}{l}{ Viscosity } & \multicolumn{3}{l|}{ pH } \\
\cline { 2 - 8 } & Plain & $\begin{array}{l}\text { Drug } \\
\text { Loaded }\end{array}$ & Plain & $\begin{array}{l}\text { Drug } \\
\text { Loaded }\end{array}$ & Plain & $\begin{array}{l}\text { Drug } \\
\text { Loaded }\end{array}$ & Plain & Drug Loaded \\
\hline ME-KHS1 & $50.60 \pm 0.04$ & $53.41 \pm 0.24$ & $63.79 \pm 0.03$ & $64.01 \pm 0.01$ & $6.18 \pm 0.01$ & $6.51 \pm 0.01$ & $1.407 \pm 0.002$ & $1.407 \pm 0.003$ \\
\hline ME-KHS2 & $77.65 \pm 0.21$ & $82.23 \pm 0.09$ & $23.96 \pm 0.05$ & $24.16 \pm 0.02$ & $5.78 \pm 0.01$ & $6.04 \pm 0.01$ & $1.384 \pm 0.002$ & $1.384 \pm 0.001$ \\
\hline ME-KHS3 & $40.60 \pm 0.07$ & $40.67 \pm 0.14$ & $55.05 \pm 0.08$ & $56.02 \pm 0.04$ & $6.16 \pm 0.01$ & $6.32 \pm 0.01$ & $1.407 \pm 0.002$ & $1.407 \pm 0.003$ \\
\hline ME-KHS4 & $68.76 \pm 0.14$ & $73.66 \pm 0.12$ & $22.15 \pm 0.06$ & $22.17 \pm 0.07$ & $5.77 \pm 0.01$ & $5.85 \pm 0.01$ & $1.384 \pm 0.001$ & $1.384 \pm 0.002$ \\
\hline & & & & & & & & \\
\hline ME-KEL1 & $37.46 \pm 0.05$ & $33.17 \pm 0.01$ & $88.00 \pm 0.19$ & $87.98 \pm 0.21$ & $4.63 \pm 0.01$ & $4.84 \pm 0.01$ & $1.407 \pm 0.005$ & $1.408 \pm 0.003$ \\
\hline ME-KEL2 & $71.24 \pm 0.08$ & $64.25 \pm 0.08$ & $41.22 \pm 0.11$ & $42.01 \pm 0.21$ & $4.22 \pm 0.01$ & $4.35 \pm 0.01$ & $1.384 \pm 0.001$ & $1.385 \pm 0.002$ \\
\hline ME-KEL3 & $34.07 \pm 0.05$ & $31.43 \pm 0.04$ & $73.88 \pm 0.15$ & $74.08 \pm 0.18$ & $4.64 \pm 0.01$ & $4.87 \pm 0.01$ & $1.407 \pm 0.001$ & $1.408 \pm 0.002$ \\
\hline ME-KEL4 & $63.35 \pm 0.12$ & $62.35 \pm 0.07$ & $35.78 \pm 0.14$ & $35.99 \pm 0.05$ & $4.19 \pm 0.01$ & $4.38 \pm 0.01$ & $1.384 \pm 0.001$ & $1.385 \pm 0.001$ \\
\hline & & & & & & & & \\
\hline ME-PL01 & $7.50 \pm 0.06$ & $6.62 \pm 0.07$ & $59.17 \pm 0.11$ & $59.19 \pm 0.11$ & $4.77 \pm 0.02$ & $4.90 \pm 0.02$ & $1.419 \pm 0.003$ & $1.419 \pm 0.002$ \\
\hline ME-PL02 & $15.86 \pm 0.03$ & $14.38 \pm 0.04$ & $57.20 \pm 0.08$ & $57.89 \pm 0.10$ & $4.47 \pm 0.04$ & $4.68 \pm 0.04$ & $1.407 \pm 0.001$ & $1.407 \pm 0.002$ \\
\hline ME-PL03 & $9.14 \pm 0.04$ & $9.01 \pm 0.03$ & $59.66 \pm 0.10$ & $60.03 \pm 0.06$ & $4.87 \pm 0.01$ & $4.91 \pm 0.01$ & $1.419 \pm 0.002$ & $1.420 \pm 0.004$ \\
\hline ME-PL04 & $18.84 \pm 0.06$ & $16.67 \pm 0.06$ & $49.88 \pm 0.17$ & $51.01 \pm 0.12$ & $4.58 \pm 0.02$ & $4.68 \pm 0.02$ & $1.407 \pm 0.002$ & $1.408 \pm 0.001$ \\
\hline
\end{tabular}


Microemulsions exhibit percolation phenomena at certain volume fractions of water. This is generally accompanied by an increase in the electrical conductivity of microemulsions, which often has been used as a method for internal structure characterization ${ }^{9,33}$. According to the percolation theory, phase transformation from W/O type structure to bicontinuous systems and then the formation of $\mathrm{O} / \mathrm{W}$ type microemulsions occur as aqueous content in the system increases ${ }^{9,14}$. The percolation threshold refers to the critical water volume fraction at which isolated droplets form infinite clusters through the emergence of bicontinuous structures ${ }^{34}$.

The IPM/(Labrasol/KEL) mixture at the oil: surfactant/co-surfactant ratio of 1:9 and the ratio of Labrasol:KEL $\left(\mathrm{K}_{\mathrm{m}}\right) 4: 1$ as weight could be diluted by water to higher than $98 \%(\mathrm{w} / \mathrm{w})$ water content and the resulting sample remained as a clear microemulsion. Also, this dilution line included the microemulsion formulation ME-KEL3 which has been presented the highest isotretinoin accumulation in pig skin in our previous study ${ }^{24}$. Therefore, in accordance with the study of Zhang\&Michniak-Kohn ${ }^{22}$, the microemulsion microstructure was studied along this water dilution line and the measured electrical conductivity values $(\kappa)$ plotted against the water content $\left(\varphi_{\mathrm{w}}\right)$ as shown in Fig. 4 . The $\kappa$ vs. $\varphi_{\mathrm{w}}$ curve showed three distinct parts, which could be fitted by linear regressions at low and high aqueous phase regions, corresponding to $\mathrm{W} / \mathrm{O}$ and $\mathrm{O} / \mathrm{W}$ microstructures. Based on these results, it can be deduced microstructure transition points from W/O to bicontinuous and from bicontinuous to $\mathrm{O} / \mathrm{W}$ were at water content of about $15 \%$ and $75 \%$, respectively.

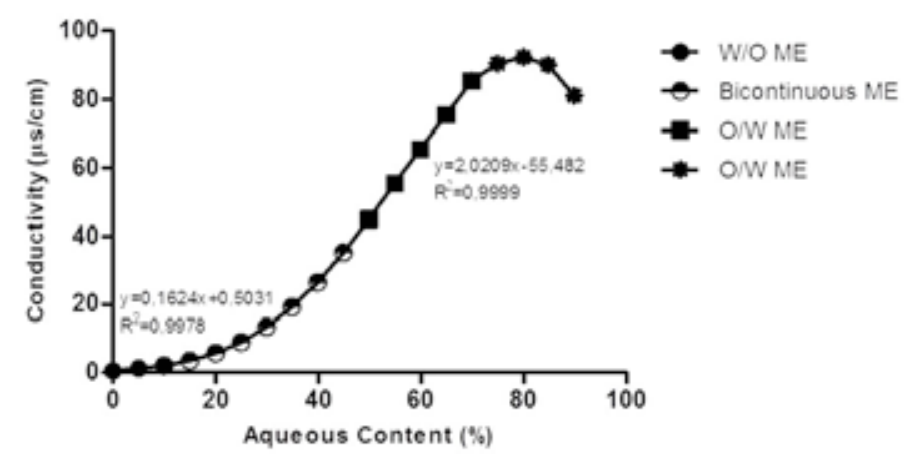

Figure 4: The plot of the microemulsion electrical conductivity to aqueous content

\section{Rheology}

The viscosity of a microemulsion is the function of the type of its components (oil, surfactant, co-surfactant and water) and their concentrations ${ }^{35}$. The viscosity of plain microemulsions was in the range of $22.15 \pm 0.06-88.00 \pm 0.19 \mathrm{cPs}$ 
(Table 2), and tended to increase as the amount of the oil and surfactant mixture in the formulation increased. This data is in accordance with the literature ${ }^{11}$. The viscosity values of isotretinoin loaded microemulsions were slightly higher than the values of unloaded formulations. All microemulsions exhibited Newtonian flow behavior (Fig. 5) due to their very low viscosity values as expected from microemulsions ${ }^{10,15,17,31}$.
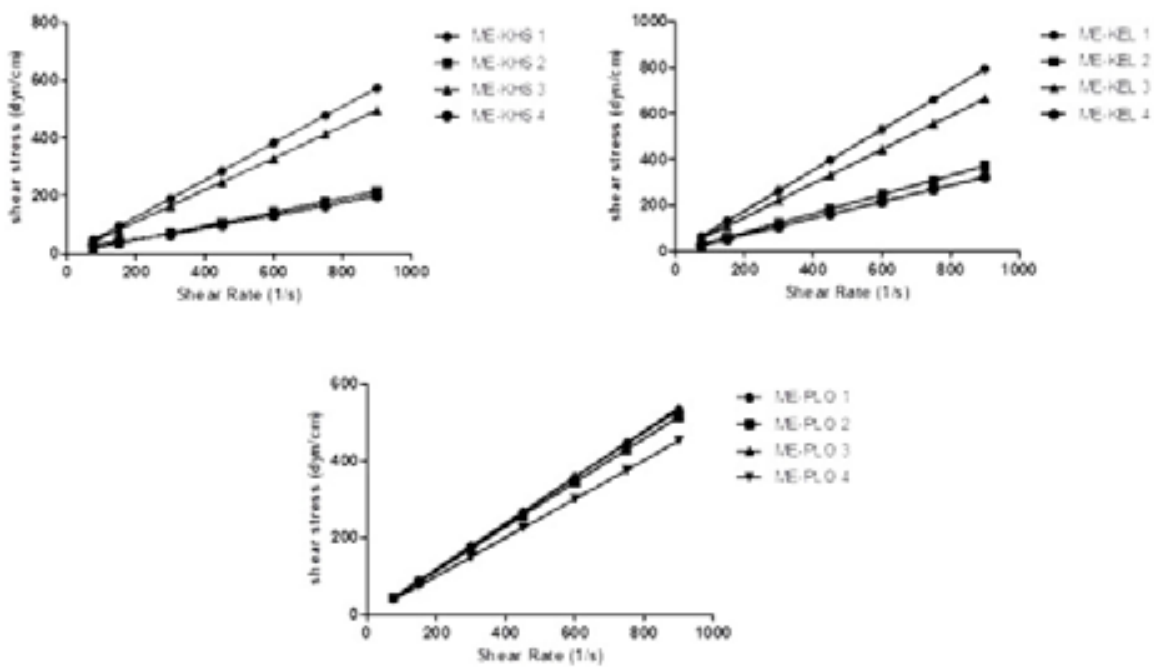

Figure 5: The plotes of shear stress versus shear rate for all of microemulsions prepared

\section{pH}

Table 2 shows the physicochemical characteristics of isotretinoin loaded microemulsions and their blank counterparts. The $\mathrm{pH}$ of the plain microemulsion formulations were between $4.19 \pm 0.01$ and $6.18 \pm 0.01$ (Table 2). Incorporation of isotretinoin slightly increased the $\mathrm{pH}$ to the range of $4.35 \pm 0.01-6.51 \pm 0.01$. The $\mathrm{pH}$ of microemulsions could be considered as suitable for cutaneous application as it has been reported that $\mathrm{pH}$ values in the range of 3 to 10 are tolerable by the skin and do not change the skin penetration of lipophilic substances ${ }^{36}$.

\section{Refractive Index}

The refractive index provides information about the dispersed and continuous phases of microemulsions and indicates their isotropic nature. The refractive index of microemulsions is expected to be close to the refractive index of the pure component forming the continuous phase ${ }^{37}$. The refractive index values of the plain and drug loaded microemulsions are demonstrated in Table 2. The refractive index values of isotretinoin loaded microemulsions $(1.384 \pm 0-1.420 \pm 0)$ were similar to their blank counterparts $(1.384 \pm 0-1.419 \pm 0)$ and confirmed the transparent nature of the formulations ${ }^{38}$. 


\section{Physical Stability}

After centrifugation at $\mathbf{1 5 0 0 0} \mathrm{rpm}$ for $30 \mathrm{~min}$ microemulsion formulations remained homogenous without any phase change such as turbidity or phase separation. Freeze thaw cycle did not result in change in droplet size or phase separation or turbidity. All formulations showed good thermodynamic stability and were taken for storage stability.

\section{Storage Stability}

The microemulsions exhibited transparency and showed no evidence on phase separation or flocculation when they were subjected to stability study at $25^{\circ} \mathrm{C} \pm 2^{\circ} \mathrm{C}$ and $60 \% \pm 5 \%$ RH for 6 months. Average droplet size of prepared microemulsion batches were measured at different time intervals and the obtained results are depicted in Fig. 6. No significant difference was observed in the PDI of microemulsions up to 6 months ( $p>0.05)$.

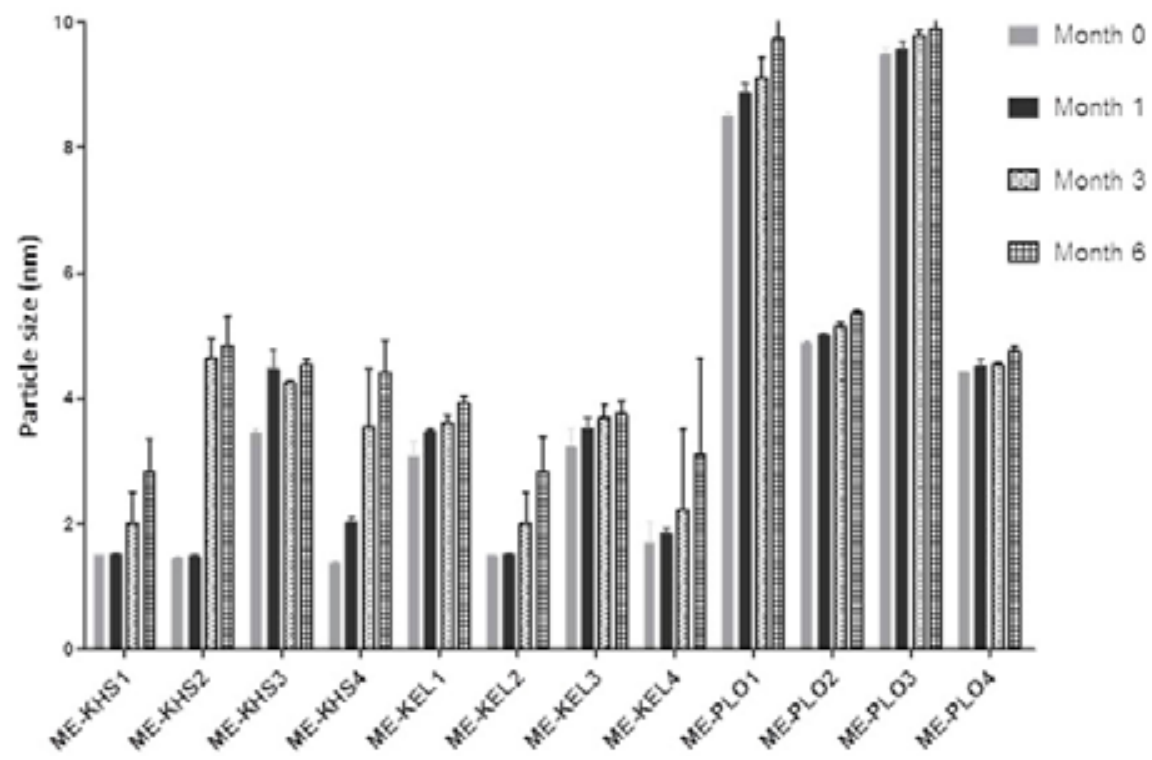

Figure 6: Droplet size and distribution of microemulsions after storage at $25 \pm 2^{\circ} \mathrm{C}$ and $60 \pm 5 \%$ RH for 6 months.

\section{CONCLUSION}

Microemulsion type colloidal carriers are one of the promising systems in skin penetration enhancement when compared with conventional formulations. Our results confirmed that the physicochemical characteristics of microemulsions are closely related to the type and ratio of the constituents and, the developed microemulsion formulations could be an alternative topical carrier to the current topical isotretinoin formulation available in the treatment of mild acne. 


\section{ACKNOWLEDGEMENT}

This research was financially supported by Istanbul University Scientific Research Projects under the number of TEZ-34200 and YADOP-7725.

\section{REFERENCES}

1. Butani, D.; Yewale, C.; Misra, A. Amphotericin B Topical Microemulsion: Formulation, Characterization and Evaluation. Colloids Surf B Biointerfaces. 2014, 116, 351-358.

2. Hosmer, J.; Reed, R.; Bentley, M. V. L.; Nornoo, A.; Lopes, L. B. Microemulsions Containing Medium-Chain Glycerides as Transdermal Delivery Systems for Hydrophilic and Hydrophobic Drugs. AAPS PharmSciTech. 2009, 10, 589-596.

3. Patel, M. R.; Patel, R. B.; Parikh, J. R. Solanki, A. B.; Patel, B. G. Investigating Effect of Microemulsion Components: In Vitro Permeation of Ketoconazole. Pharm Dev Technol. 2o11, $16,250-258$.

4. Trotta, M.; Ugazio, E.; Peira, E.; Pulitano, C. Influence of Ion Pairing on Topical Delivery of Retinoic Acid from Microemulsions. J Control Release. 2oo3, 86, 315-321.

5. Patravale, V. B.; Date, A. A. Microemulsions: Pharmaceutical Applications. In Microemulsions Background, New Concepts, Applications, Perspectives, 1st ed.; Stubenrauch, C., Eds.; Blackwell Publishing: UK, 2009; pp 259-301.

6. Sintov, A. C.; Shapiro, L. New Microemulsion Vehicle Facilitates Percutaneous Penetration In Vitro and Cutaneous Drug Bioavailability In Vivo. J Control Release. 2004, 95, 173-183.

7. Teichmann, A.; Heuschkel, S.; Jacobi, U.; Presse, G.; Neubert, R. H. H.; Sterry, W.; Lademann, J. Comparison of Stratum Corneum Penetration and Localization of a Lipophilic Model Drug Applied in an O/W Microemulsion and an Amphiphilic Cream. Eur J Pharm Biopharm. 2007, 67, 699-706.

8. Baroli, B.; Lo' pez-Quintela, M. A.; Delgado-Charro, M.B.; Fadda, A. M., Blanco-Me'ndez, J. Microemulsions for Topical Delivery of 8-methoxsalen. J Control Release. 2ooo, 69, 209-218.

9. Lopes, L. B. Overcoming the Cutaneous Barrier with Microemulsions. Pharmaceutics. 2014, 6, 52-77.

10. Talegaonkar, S.; Azeem, A.; Ahmad, F. J. Khar, R. K.; Pathan, S. A. ; Khan, Z. I. Microemulsions: A Novel Approach to Enhanced Drug Delivery. Recent Pat Drug Deliv Formul. 2oo8, $2,238-257$.

11. Ngawhirunpat, T.; Worachun, N.; Opanasopit, P.; Rojanarata, T.; Panomsuk, S. Cremophor RH40-PEG 400 Microemulsions as Transdermal Drug Delivery Carrier for Ketoprofen. Pharm Dev Technol. 2013, 18, 798-803.

12. Pandey, A.; Mittal, A.; Chauhan, N.; Alam, S. Role of Surfactants as Penetration Enhancer in Transdermal Drug Delivery System. J Mol Pharm Org Process Res. [Online early access]. DOI: 10.4172/2329-9053.1000113. Published Online: May 15, 2014.

13. Djekic, L.; Primorac, M. The Influence of Cosurfactants and Oils on the Formation of Pharmaceutical Microemulsions Based on PEG-8 Caprylic/Capric Glycerides. Int J Pharm. 2oo8, 352, 231-239.

14. Ge, S.; Lin, Y.; Lu, H.; Li, Q.; He, J.; Chen, B.; Wu, C.; Xu, Y. Percutaneous Delivery of Econazole Using Microemulsion as Vehicle: Formulation, Evaluation and Vesicle-Skin Interaction. Int $J$ Pharm. 2014, 465, 120-131. 
15. Kogan, A.; Garti, N. Microemulsions as Transdermal Drug Delivery Vehicles. Adv Colloid Interface Sci. 2006, 123-126, 369-385.

16. Ma, H.; Yu, M.; Lei, M.; Tan, F.; Li, N. A Novel Topical Targeting System of Caffeine Microemulsion for Inhibiting UVB-Induced Skin Tumor: Characterization, Optimization, and Evaluation. AAPS PharmSciTech. 2015, 16, 905-913.

17. Boonme, P.; Boonthongchuay, C.; Wongpoowarak, W.; Amnuaikit, T. Evaluation of Nicotinamide Microemulsion on the Skin Penetration Enhancement. Pharm Dev Technol. 2016, 21, 116-120.

18. Vlaia, L.; Olariu, I.; Coneac, G.; Mut, A. M.; Popoiu, C.; Corina, S.; Anghel, D. F.; Maxim, M. E. Kalas, S.; Vlaia, V. Development of Microemulsion-Loaded Hydrogel Formulations for Topical Delivery of Metoprolol Tartrate: Physicochemical Characterization and Ex Vivo Evaluation. Farmacia. 2016, 64, 901-913.

19. Zhao, L.; Wang, Y.; Zhai, Y.; Wang, Z.; Liu, J.; Zhai, G. Ropivacaine Loaded Microemulsion and Microemulsion-Based Gel for Transdermal Delivery: Preparation, Optimization, and Evaluation. Int J Pharm. 2014, 477, 47-56.

20. Hathout, R. M.; Woodman, T. J.; Mansour, S.; Mortada, N. D.; Geneidi, A. S.; Guy, R. H. Microemulsion Formulations for the Transdermal Delivery of Testosterone. Eur J Pharm Sci. 2010, $40,188-196$.

21. Kuntsche, J.; Horst, J. C.; Bunjes, H. Cryogenic Transmission Electron Microscopy (cryoTEM) for Studying the Morphology of Colloidal Drug Delivery Systems. Int J Pharm. 2o11, 417, 120-137.

22. Zhang, J.; Michniak-Kohn, B. Investigation of Microemulsion Microstructures and Their Relationship to Transdermal Permeation of Model Drugs: Ketoprofen, Lidocaine, and Caffeine. Int J Pharm. 2011, 421, 34-44.

23. International Conference on Harmonisation (ICH) of Technical Requirements for Registration of Pharmaceuticals for human use. Stability testing of new drug substances and products. Q1 A (R2). 2005.

24. Gürbüz A.; Özhan G.; Güngör S.; Erdal M.S. Colloidal Carriers of Isotretinoin for Topical Acne Treatment: Skin Uptake, ATR-FTIR and In Vitro Cytotoxicity Studies. Arch Dermatol Res. 2015, 307, 607-615.

25. Sintov, A. C. Transdermal Delivery of Curcumin via Microemulsion. Int J Pharm. 2015 , 481, 97-103.

26. Ita, K. Progress in the Use of Microemulsions for Transdermal and Dermal Drug Delivery. Pharm Dev Technol. [Online early access]. DOI: 10.3109/10837450.2016.1148722. Published Online: February 26, 2016.

27. Heuschkel, S.; Goebel, A.; Neubert, R. H. H. Microemulsion-Modern Colloidal Carrier for Dermal and Transdermal Drug Delivery. J Pharm Sci. 20o8, 97, 603-631.

28. Aggarwal, N.; Goindi, S.; Khurana, R. Formulation, Characterization and Evaluation of an Optimized Microemulsion Formulation of Griseofulvin for Topical Application. Colloids Surf B Biointerfaces. 2013, 105, 158-166.

29. Biruss, B.; Kählig, H.; Valenta, C. Evaluation of an Eucalyptus Oil Containing Topical Drug Delivery System for Selected Steroid Hormones. Int J Pharm. 2007, 328, 142-151.

30. Müller-Goymann, C. C. Physicochemical Characterization of Colloidal Drug Delivery Sys- 
tems such as Reverse Micelles, Vesicles, Liquid Crystals and Nanoparticles for Topical Administration. Eur J Pharm Biopharm. 2004, 58, 343-356.

31. Leanpolchareanchai, J.; Padois, K.; Falson, F.; Bavovada, R.; Pithayanukul, P. Microemulsion System for Topical Delivery of Thai Mango Seed Kernel Extract: Development, Physicochemical Characterisation and Ex Vivo Skin Permeation Studies. Molecules. 2014, 19, 1710717129 .

32. Lawrence, M. J.; Rees, G. D. Microemulsion-Based Media as Novel Drug Delivery Systems. Adv Drug Deliv Rev. 2012, 64, 175-193.

33. Djordjevic, L.; Primorac, M.; Stupar, M.; Krajisnik, D. Characterization of Caprylocaproyl Macrogolglycerides Based Microemulsion Drug Delivery Vehicles for an Amphiphilic Drug. Int J Pharm. 2004, 271, 11-19.

34. Mahrhauser, D. S.; Kählig, H.; Partyka-Jankowska, E.; Peterlik, H.; Binder, L.; Kwizda, K.; Valenta, C. Investigation of Microemulsion Microstructure and Its Impact on Skin Delivery of Flufenamic Acid. Int J Pharm. 2015, 490, 292-297.

35. Mahrhauser, D.; Hoppel, M.; Schöll, J.; Binder, L.; Kählig, H.; Valenta, C. Simultaneous Analysis of Skin Penetration of Surfactant and Active Drug From Fluorosurfactant-Based Microemulsions. Eur J Pharm Biopharm. 2014, 88, 34-39.

36. Bettoni, C. C.; Felippi, C. C.; de Andrade, C.; Raffin, R. P.; Jager, A.; Guterres, S. S.; Costal, T. D. Isotretinoin-Loaded Nanocapsules: Stability and Cutaneous Penetration by Tape Stripping in Human and Pig Skin. J Biomed Nanotechnol. 2012, 8, 258-271.

37. Alam, S.; Iqbal, Z.; Ali, A.; Khar, R. K.; Ahmad, F. J.;Akhter, S., Talegaonkar, S. Microemulsion as a Potential Transdermal Carrier for Poorly Water Soluble Antifungal Drug Itraconazole. J Disper Sci Technol. 2009, 31, 84-94.

38. Patel, R. B.; Patel, M. R.; Bhatt, K. K.; Patel, B. G. Formulation Consideration and Characterization of Microemulsion Drug Delivery System for Transnasal Administration of Carbamazepine. Bulletin of Faculty of Pharmacy, Cairo University. 2013, 54, 107-274.

(Received o7 February 2017; accepted 25 February 2017) 